\title{
Le numérique, pour coopérer en formation à distance
}

Digital technology, to cooperate in distance learning

Tecnologías digitales para la cooperación en educación a distancia

Nicole Racette et Marie-Pierre Bourdages-Sylvain

\section{OpenEdition}

\section{Journals}

Édition électronique

URL : http://journals.openedition.org/ctd/634

DOI : $10.4000 /$ ctd. 634

ISSN : 2491-1437

Éditeur

Chaire Unesco Pratiques émergentes en technologies et communication pour le développement

Référence électronique

Nicole Racette et Marie-Pierre Bourdages-Sylvain, «Le numérique, pour coopérer en formation à distance », Communication, technologies et développement [En ligne], 6 | 2018, mis en ligne le 18 décembre 2018, consulté le 30 avril 2019. URL : http://journals.openedition.org/ctd/634 ; DOI : $10.4000 /$ ctd. 634

Ce document a été généré automatiquement le 30 avril 2019

Communication, technologies et développement 


\title{
Le numérique, pour coopérer en formation à distance
}

\author{
Digital technology, to cooperate in distance learning \\ Tecnologías digitales para la cooperación en educación a distancia
}

Nicole Racette et Marie-Pierre Bourdages-Sylvain

Bien que le numérique ait permis le déploiement des cours à grande échelle, il a également mis à l'épreuve les pratiques d'enseignement, où les tâches de conception, de diffusion des cours, d'encadrement des étudiants et de suivi du bon fonctionnement des cours ne sont plus totalement du ressort de l'enseignant, mais plutôt réparties entre différents spécialistes (Guillemet, 1993). Malgré l'engouement des apprenants pour la formation à distance (FAD) (Dilworth et al., 2012; Klisc, McGill et Hobbs, 2012 ; Nworie, Haughton et Oprandi, 2012; Spires, Wiebe, Young, Hollebrands et Lee, 2012), ces apprenants ont plus de difficultés à persévérer et à bien réussir leurs cours qu'en formation en face à face (Cho, 2010; Zha et Ottendorfer, 2011). Ces performances plus faibles peuvent être attribuables à des caractéristiques de la clientèle desservie (temps partiel, conciliation travail-études-famille, etc.), mais aussi à d'autres causes, dont la qualité des cours et la qualité de l'encadrement. Selon Decamps et Depover (2011), les responsables de première ligne affectés à ces apprenants sont les équipes de conception (enseignants, concepteurs et conseillers pédagogiques) et les équipes d'encadrement (tuteurs, correcteurs, encadrants ou ressources pédagogiques) qui travaillent à distance les unes des autres: les équipes d'encadrement travaillant à partir de leur domicile respectif et les équipes de conception, à partir des établissements (Racette, Poellhuber et Bourdages-Sylvain, soumis en octobre 2017). Ainsi, les cours sont normalement conçus par des équipes de conception, interprétés par les équipes d'encadrement et utilisés par les étudiants pour apprendre les concepts qu'on y enseigne (Bertin et Narcy-Combes, 2012 ; Comas-Quinn, de los Arcos et Mardomingo, 2012). Selon Racette, Poellhuber et Bourdages-Sylvain (2017), parce que les équipes de conception délèguent l'encadrement des étudiants aux équipes d'encadrement, ces dernières s'attendent à être informées des particularités que contiennent les cours ainsi que des attentes en termes d'encadrement. D'un autre côté, les équipes de conception s'attendent à être informées de ce qui se passe 
dans leurs cours, soit des aspects appréciés et moins appréciés par les étudiants ainsi que des irrégularités décelées. Mais surtout, ils s'attendent à ce que les tuteurs suivent les directives qu'ils ont reçues. Puisqu'il s'agit de télétravail, pour que ces échanges puissent se faire, un climat de coopération est nécessaire dans les communications basées principalement sur les outils électroniques. Un manque de coopération est susceptible d'entrainer une perte de sens dans ce travail de production de cours, pouvant affecter la qualité des cours offerts.

2 Dans une recherche financée par le Conseil de recherche en sciences humaines (CRSH), nous avons abordé la question suivante : comment le numérique contribue-t-il ou limitet-il la coopération entre les équipes de conception et les équipes d'encadrement? Nous identifions tout d'abord les paramètres de la coopération en milieu de travail à travers la théorie de l'échange social et, selon le classement de Walder (2016), différentes catégories d'outils numériques pour communiquer. Nous présentons ensuite la méthodologie et les résultats de cette recherche sur la qualité des communications et des relations, les outils de communication utilisés et des recommandations pour augmenter la coopération entre les équipes de conception et les équipes d'encadrement.

\section{La théorie de l'échange social}

3 Selon Tsai et Cheng (2012) et Filipowski, Kazienko et Brodka (2012), la théorie de l'échange social compte parmi les paradigmes les plus influents pour comprendre le comportement de coopération au travail. Elle met en évidence la notion de récompenses qui ne se monnaient pas, soit une reconnaissance de l'apport de chacun, les encouragements et une appréciation du travail fait (Bock et Kim, 2012). Selon cette théorie, la personne se sent tenue d'aller au-delà des tâches prescrites lorsqu'elle reçoit ces récompenses intrinsèques. L'échange social permet d'engendrer des sentiments d'obligation personnelle, de gratitude et de confiance (Filipowski et al., 2012). Cette théorie repose elle-même sur une théorie de la justice. Dès qu'une des deux parties n'offre pas, selon la perception de l'autre, des conditions de réciprocité, elle perd tout le potentiel de coopération de l'autre (Nworie et al., 2012). Très vite, ces personnes développent des sentiments d'anomie (chaos dû à l'absence de règles), d'absurdité, d'étrangeté et d'isolement (Filipowski et al., 2012). En corollaire, si les gens sentent que leur environnement de travail est empreint de loyauté, ils manifestent un comportement élevé de partage des connaissances et de coopération (Tsai et Cheng, 2012). Il doit y avoir équilibre dans les bénéfices obtenus pour que l'échange social se manifeste et qu'une coopération s'installe.

\section{L'impact des outils numériques sur le travail}

4 Le numérique transforme le travail et les relations entre les individus pour faire autrement ensemble, ce qui est d'autant plus vrai avec le télétravail. En FAD, le télétravail est imposé aux tuteurs qui ne bénéficient pas d'un espace de travail à l'établissement. Contrairement au travail qui se fait à l'établissement, le travail à distance est moins facilement mesurable et contrôlable (Boboc et Taboy, 2014). De plus, une mauvaise utilisation des outils numériques dans ce contexte peut produire des résultats contreproductifs, soit une fragmentation des tâches, une surcharge d'information, une 
dépersonnalisation des relations, du stress et l'aplatissement des hiérarchies, pour ne nommer que ceux-là (Boboc et Taboy, 2014; Casasus, 2015). Short, Williams et Christie (1976, p. 530) parlent de présence sociale dans les communications, qu'ils définissent ainsi : «the degree of salience of the other person in a mediated communication and the consequent salience of their interpersonnal interactions ». Il s'agit d'une perception émotive d'appartenance qui est importante dans toute forme de télécommunication (Wei, Chen et Kinshuk, 2012). Ainsi, une mauvaise utilisation des outils électroniques pour transmettre des directives hiérarchiques risque de déshumaniser les relations entre les salariés lorsque ces directives ne peuvent pas être discutées ni questionnées. Elles risquent d'être mal interprétées, induisant de la confusion (Casasus, 2015). Selon Walder (2016), nous pouvons classer les outils de communication utilisés par les équipes de conception et les équipes d'encadrement selon qu'ils servent au transfert de connaissances (informer), aux échanges (discuter, argumenter) ou au développement des connaissances (former).

Les wikis et les capsules vidéo constituent de bons outils de transfert des connaissances, lorsqu'ils visent à informer et non pas à échanger des idées, à statuer sur un point ni à prendre une décision. Les outils d'échange, selon Walder (2016, p. 14), portent sur « les diapositives, les présentations visuelles, les télévoteurs, les forums de discussion et les visioconférences ». Ils permettent l'écoute, la réflexion, le débat, la collaboration ainsi qu'une perspective sociale. Les réunions en ligne, avec l'audio, le son et la caméra Web, induisent la quasi-présence, grâce à des logiciels tels que Skype, Adobe Connect, Via ou Enjeu (Kaattari, 2011), ce qui est en lien avec la théorie de la richesse des médias, selon laquelle plus un médium est interactif et riche sur le plan médiatique, plus il est susceptible de véhiculer des indices non verbaux qui permettent d'enrichir la communication, et, ultimement, de véhiculer la présence sociale. Les échanges audiovisuels permettent des communications plus naturelles et sont plus susceptibles de véhiculer la présence sociale, mais demandent beaucoup plus de préparation, parce que l'improvisation y a peu de place et les problèmes techniques sont fréquents, mais aussi parce que la qualité de la mise en page, de la lisibilité des textes et de la transmission sonore et visuelle est essentielle pour garder l'intérêt et assurer la compréhension des éléments présentés (Loisier, 2009). Les réseaux sociaux mettent en contact des personnes, des organisations et des entreprises, tels que Facebook (le 2e site le plus populaire au monde), Linkedln, Ning et MySpace (Kaattari, 2011). Toutefois, par rapport à ces outils d'échange, «les pratiques de consultation sont plus répandues que les pratiques de contribution » (Boboc et Taboy, 2014, p. 4). Selon Walder (2016), les courriels constituent un outil d'échange. Toutefois, selon Loisier (2009), ils constituent un bon moyen pour transmettre des informations, mais un piètre moyen pour échanger, étant donné qu'il est difficile d'y créer un climat de confiance et d'empathie. Selon ce même auteur, les courriels permettent de prendre le temps nécessaire pour s'exprimer, contrairement à la téléphonie par exemple, mais ils ouvrent souvent la porte à une familiarité inappropriée, à l'interprétation erronée, à la nécessité d'utiliser plusieurs va-et-vient de courriels pour atteindre la compréhension désirée. D'autres auteurs (Fernández-Toro et Furnborough, 2014 ; Robinson, Centifanti, Brewer et Holyoak, 2015) expliquent que les communications à distance et en asynchrone sont souvent peu personnalisées et trop générales pour inciter les usagers à coopérer entre eux. Relativement au classement de Walder (2016, p. 15), « les modèles 3D, les logiciels et les cartes conceptuelles » constituent des outils de développement des connaissances, parce qu'ils permettent d'analyser des résultats, de 
faire des liens, de qualifier l'apprenant, et ce, dans un temps restreint. Les blogues sont de plus en plus populaires. Ils sont principalement utilisés pour le développement des connaissances, pouvant contenir du texte, des photos, des vidéos avec audio ainsi que des liens. Mais comment utilise-t-on ces outils de transfert, d'échange et de développement des connaissances pour lier le travail de conception de cours et le travail d'encadrement des étudiants?

\section{La méthodologie}

Cette recherche, de type exploratoire, prend la forme d'une étude de cas multiples (Creswell, 2012) sur les moyens utilisés par les équipes de conception et les équipes d'encadrement pour coopérer, et ce, dans trois établissements d'enseignement canadiens et un européen: la TÉLUQ, la Faculté d'éducation permanente de l'Université de Montréal, le Cégep@distance et l'Enseignement à distance de la Fédération WallonieBruxelles. La collecte de données repose sur une méthode mixte: 22 entrevues individuelles auprès de tuteurs, 14 auprès de membres d'équipes de conception et 8 auprès de directeurs administratifs, des commentaires reçus du personnel par rapport à une vidéo présentant les résultats des entrevues individuelles ainsi que 8 entrevues de groupe. Les verbatim réalisés à partir des entrevues enregistrées ont été codés dans QDA Miner, avec un accord interjuges dépassant les $80 \%$. Les verbatim ont tous été épurés, c'est-à-dire que l'écriture a été adaptée au langage écrit plutôt qu'oral. Ils ont été séparés en unité de sens, chacun de ces extraits ayant été rendu autonome afin d'en garder le sens, et ce, même lorsque lu hors contexte. Les citations des équipes d'encadrement, soit les tuteurs, sont identifiées par un (T), celles des équipes de conception par un (C) et celles des directeurs administratifs par un (D).

\section{La qualité des communications et des relations}

7 Le tableau 1 présente la fréquence des codes, positifs et négatifs, en termes d'extraits ainsi que de nombre de répondants, sur l'information transmise (qui ne demande aucune réponse), les réponses obtenues aux demandes d'informations, le suivi des directives, l'attitude proactive des équipes de conception ou des équipes d'encadrement, la reconnaissance du travail de chacun, le partenariat perçu par chacun et, plus précisément, sur l'absence de relation constatée entre les équipes de conception et les équipes d'encadrement.

Tableau 1 : Fréquence des codes sur la qualité de la communication entre équipes de conception et équipes d'encadrement

\begin{tabular}{|l|l|l|}
\hline \multicolumn{2}{|c|}{} & N = 4 établissements \\
\cline { 2 - 3 } & $\begin{array}{l}\text { Nombre d'extraits } \mathrm{N}=1286 \\
(\%)\end{array}$ & $\begin{array}{l}\text { Nombre de répondants } \mathrm{N}=44 \\
(\%)\end{array}$ \\
\hline $\begin{array}{l}\text { Informations transmises } \\
\text { (ne demandant pas de réponse) }\end{array}$ & $205(15,9 \%)$ & $41(93,2 \%)$ \\
\hline
\end{tabular}




\begin{tabular}{|c|c|c|}
\hline Positif & $148(11,5 \%)$ & $36(81,8 \%)$ \\
\hline Négatif & $57(4,4 \%)$ & $22(50 \%))$ \\
\hline $\begin{array}{l}\text { Réponse à la demande } \\
\text { d'information }\end{array}$ & $85(6,6 \%)$ & $21(47,7 \%)$ \\
\hline Positif & $51(4 \%)$ & $20(45,5 \%)$ \\
\hline Négatif & $34(2,6 \%)$ & $15(34,1 \%)$ \\
\hline Suivi des directives & $73(5,7 \%)$ & $20(45,5 \%)$ \\
\hline Positif & $31(2,4 \%)$ & $15(34,1 \%)$ \\
\hline Négatif & $42(3,3 \%)$ & $18(40,9 \%)$ \\
\hline Attitude proactive & $99(7,7 \%)$ & $29(65,9 \%)$ \\
\hline Positif & $59(4,6 \%)$ & $22(50 \%)$ \\
\hline Négatif & $40(3,1 \%)$ & $17(38,6 \%)$ \\
\hline Reconnaissance du travail fait & $102(7,9 \%)$ & $25(56,8 \%)$ \\
\hline Positif & $60(4,7 \%)$ & $19(43,2 \%)$ \\
\hline Négatif & $42(3,3 \%)$ & $12(27,3 \%)$ \\
\hline Perception de partenariat & $722(56,1 \%)$ & $44(100 \%)$ \\
\hline Positif & $456(35,5 \%)$ & $42(95 \%)$ \\
\hline Négatif & $266(20,7 \%)$ & $37(84,1 \%)$ \\
\hline Absence de relation & 39 (3\%) & $15(34,1 \%)$ \\
\hline
\end{tabular}

En termes d'importance, les extraits codés s'avèrent soit très positifs, soit très négatifs. Plusieurs répondants qui ont parlé d'un thème en ont parlé à la fois positivement et négativement, ce qui peut s'expliquer par le fait que chaque tuteur peut devoir travailler avec plusieurs équipes de conception, et vice-versa, chacun ayant ses façons de faire, ses bons et ses mauvais côtés. Trois thèmes importants ressortent de ce tableau: le partenariat, où $100 \%$ des répondants ont émis des commentaires, ce qui constituait un thème central à notre étude, le suivi des directives, où le nombre d'extraits et le nombre de répondants négatifs ont été plus nombreux que ceux positifs et, finalement, l'absence de relation exprimée par $34 \%$ des répondants, constituant une entrave importante à la coopération. 


\section{Le partenariat}

9 Ainsi, $56,1 \%$ des extraits portent sur le partenariat (100\% des répondants), où $95 \%$ des répondants en ont parlé positivement, provenant tant des équipes de conception que des équipes d'encadrement : « Récemment, un des professeurs a pris le temps de consulter les tuteurs. Depuis ce temps, on a beaucoup plus de contacts ; c'est plus positif et ça va très bien » $(\mathrm{T})$. «J'ai eu beaucoup d'expériences avec des professeurs extraordinaires, très humains, avec lesquels je me suis très bien entendu » $(\mathrm{T})$. « Généralement, ça va bien, J'ai une bonne relation avec mes tuteurs. Je suis content de travailler avec eux» (C). Toutefois, $84,1 \%$ des répondants avaient aussi des reproches à faire sur le partenariat : "Il faut dire qu'il y a peu d'interactions naturelles entre les professeurs et les tuteurs " (C). «C'est difficile pour moi, de coopérer avec les professeurs, parce que dans une même année, je peux compter les contacts que j'ai avec eux sur les doigts d'une main » (T). «Je ne comprends pas pourquoi les professeurs ne travaillent pas avec les tuteurs» (T). « Pour moi, le travail en partenariat avec les tuteurs, ce n'est pas nécessairement facile. Mon équipe tutorale peut compter 1-2-10 ou, même, 15 tuteurs » (C).

\section{Le suivi des directives}

Quant au suivi des directives, nous constatons moins d'éléments positifs que négatifs (2,4\% contre $3,3 \%$ des extraits et $34,1 \%$ contre $40,9 \%$ des répondants) ainsi qu'une grande importance des extraits négatifs en termes d'impact sur l'encadrement. Entre autres, les membres des équipes de conception sont préoccupés par le fait qu'il soit difficile de faire un suivi sur l'application des directives: «Je ne sais pas si les directives pédagogiques sont suivies par les tuteurs, parce qu'en fait, il est très difficile de savoir ce que font les tuteurs avec leurs étudiants » (C). «Si le cours va de plus en plus mal, on ne le sait pas» (C). Ces constats confirment le problème du contrôle des employés dans le télétravail, tel que défini par Boboc et Taboy (2014). Nous constatons que, loin d'aller audelà des tâches prescrites, ces équipes ne suivent majoritairement pas les directives. « Les tuteurs n'ont pas de préoccupation par rapport aux équipes de conception» (T). "Contrairement à la directive, je ne fais pas d'appel téléphonique au début du cours " (T). « Ils voulaient que je planifie des dates différentes pour 10 cours différents, pour 200 étudiants. Non je ne vais pas faire ça » (T). Selon les répondants, certains tuteurs se positionnent volontairement à l'encontre des directives reçues, alors que d'autres font une mauvaise interprétation des directives, ce qui constitue une entrave importante à la coopération, telle que définie par Filipowski et al. (2012).

\section{L'absence de relation}

11 L'absence de relations entre les équipes de conception et les tuteurs a été évoquée par $34,1 \%$ des répondants. «Il y a des cours où il n'y a pas de relations» (C). « Il y a un professeur dans mon cours, mais je ne l'ai jamais vu. Je n'ai jamais eu de contacts avec lui. Il n'a jamais répondu à mes courriels » $(\mathrm{T})$. «Je ne contacte pas certains des tuteurs assignés à mes cours, parce que par courriel, ils ne répondent pas. Ils m'ont signalé qu'ils ne voulaient pas participer en équipe, qu'ils ne voulaient pas se déplacer, qu'ils étaient très contents de la situation actuelle » (C). «L'équipe d'encadrement, ce sont plus de 100 
personnes qui travaillent avec nous dans notre organisation et que nous ne voyons jamais. Nous ne leur parlons jamais. Ce n'est pas normal » (C). Ces résultats montrent un inconfort important, surtout exprimé par les tuteurs qui se voient souvent laissés à euxmêmes pour répondre à des situations problématiques dans les cours.

\section{Les outils de communication utilisés}

12

Lacommunicationécritedemeurelemoyenprivilégiéparleséquipesd'encadrementetleséquipes de conception pour communiquer (Robinson et al., 2015), ce que confirment nos résultats. Cette communication écrite se fait surtout par courriel, malgré l'avènement des technologies de plus en plus performantes pour communiquer en synchrone (Depover et Quintin, 2011). Les enseignants les apprécient tant pour leur rapidité que pour leur accessibilité. «Lorsqu'il y a des directives ou beaucoup d'informations à divulguer, c'est plus rapide par courriel» (c). Toutefois, les répondants en soulignent certaines limites, soit les mauvaises interprétations possibles de leur contenu ainsi qu'une source potentielle de différends. " Ça crée des conflits du fait que la communication ne se fait pas en face à face, mais par courriels » (C). « Un courriel formel et autoritaire, c'est vraiment désagréable " $(\mathrm{T})$. Les réponses aux demandes reçues sont parfois incomplètes. "La plupart des enseignants répondent rapidement à mes courriels, mais les informations fournies sont parfois incomplètes » $(\mathrm{T})$. D'autres encore dénoncent l'absence de réponse de certains intervenants, qui peut sérieusement entraver la coopération. Dans ce cas, les courriels ne constituent pas un outil d'échange. Lorsqu'il s'agit du principal moyen de communication utilisé, ils peuvent constituer une limite importante à la coopération, devant être considérée plutôt comme un outil de transfert des connaissances, nous semble-t-il.

Tous les établissements à l'étude mettaient des forums de discussion et des visioconférences à la disposition des équipes de conception et des équipes d'encadrement pour échanger entre elles ainsi qu'entre pairs. Toutefois, ils étaient très peu utilisés : « La visioconférence a été expérimentée par un professeur. Mais elle est très très peu utilisée. On a 160 cours et je pense que ce n'est pas utilisé du tout »(C). La nouveauté des outils et la méconnaissance de leur fonctionnement sont autant de facteurs mis en cause dans leur faible utilisation, tout comme la culture organisationnelle ainsi que le manque de coopération initial entre ces équipes. «Le problème, ce n'est pas qu'ils n'existent pas ou qu'on n'y ait pas accès, c'est qu'il n'y a pas de pratiques organisationnelles où on est en contact tout le temps par ces moyens-là » $(\mathrm{T})$. «Les enseignants ont le sentiment que le cours leur appartient et qu'ils n'ont pas de comptes à rendre aux tuteurs » (C).

Dans les quatre établissements, des rencontres sont normalement organisées en présentiel pour former à l'encadrement des nouveaux cours. Bien qu'il semble que ce soit rare, les répondants nous ont signalé que des tuteurs sont parfois embauchés sans recevoir aucune formation sur le cours ni sur les pratiques d'encadrement. Pourtant, l'importance inédite que prend l'encadrement en FAD nécessite de plus en plus une formation pointue selon plusieurs auteurs (Berrouk et Jaillet, 2010; Depover, 2013; Depover, Komis et Karsenti, 2012 ; Glikman, 2014 ; Sarpentier, 2015). Selon les répondants, des outils de développement des connaissances pourraient être utilisés davantage pour former les tuteurs sur le contenu des cours dans lesquels ils interviennent ainsi que sur les outils technologiques qu'ils auront à utiliser avec les étudiants, en plus d'être formés 
sur les aspects pédagogiques et socioaffectifs. «Ce qui manque, c'est plus d'informations et de formations, pas sur le cours en tant que tel, mais sur toute la procédure, afin d'être plus efficace » $(\mathrm{T})$.

\section{Pour plus de coopération}

15 Afin de remédier à la situation, plusieurs répondants ont émis le souhait que des rencontres annuelles en face à face soient organisées, afin que les équipes de conception et d'encadrement puissent se rencontrer, échanger et tisser des liens, ce qui faciliterait les rencontres virtuelles par la suite et une plus grande coopération. "C'est toujours mieux en face à face. On voit ainsi l'énergie de la personne. Ce n'est pas comme sur un écran » (T). "Quand on met les enseignants et les tuteurs ensemble, il y a toujours quelque chose d'intéressant qui en ressort » (T). Boboc et Taboy (2014, p. 3) constatent que «les communautés les plus actives en ligne sont celles qui préexistaient dans la " vraie vie ", ce que confirme nos résultats. Les répondants réclament des échanges moins formels et, dans le cas des tuteurs, des outils de développement des connaissances pour que les tuteurs puissent prendre pleinement la place qu'on attend d'eux. Les blogues, les wikis et les diapositives en ligne (en format PowerPoint ou Prezi) constituent différentes façons de former le personnel. La technologie SlideShare permet aussi de partager des diaporamas en ligne. Adobe Presenter peut être utilisé pour ajouter une narration aux diapositives, des vidéos, des jeux-questionnaires, etc. (Kaattari, 2011). Les vidéos en ligne sont aussi utilisées pour donner de la formation, comme ce qu'on trouve sur YouTube, Adobe Flash Player et Vimeo. Les vidéocaptures les plus populaires sont Jing et Camtasia (Kaattari, 2011). Les webinaires et les cyberconférences, tels que GoToWebinar, WebEx, Adobe Connect, Ready Talk, Centra, Elluminate, First Class et Wimba, relient les participants en temps réel tout en permettant la consultation des enregistrements par la suite. Ils servent normalement à donner de la formation tout en facilitant les échanges entre le formateur et les participants, par le clavardage, les enquêtes et les sondages. Mais aucun de ces outils de développement des connaissances ne semble utilisé pour former les tuteurs qui travaillent éloignés de l'établissement et des équipes de conception. Pour l'instant, il semble que le personnel réponde plutôt aux urgences en fonction des courriels reçus. Ils sont davantage réactifs que proactifs, ce qui déçoit surtout les tuteurs, puisque ce sont eux qui souffrent le plus des problèmes de communication observés, se retrouvant souvent désarmés pour bien faire leur travail envers les étudiants. Ainsi, audelà de la coopération, nos résultats montrent que dans plusieurs cas, il n'y a même pas un bon transfert d'informations.

16 Les communications entre les équipes de conception et les équipes d'encadrement, se faisant presque exclusivement à distance via les courriels, à l'instar des conclusions de Loisier (2009), ne permettent pas de créer un climat de confiance et d'empathie ni un échange social qui permettrait d'engendrer des sentiments d'obligation personnelle et une réciprocité favorisant la coopération (Bock et Kim, 2012 ; Filipowki et al., 2012). Les équipes de conception et les équipes d'encadrement doivent donc être formées à choisir les bons outils en fonction des buts poursuivis (transfert d'informations, échange ou formation), et savoir comment les utiliser. Mais pourquoi n'est-ce pas déjà fait ? Cette recherche portait sur des organisations disposant d'une culture organisationnelle et d'enjeux de relations de travail distincts et, pourtant, toutes ces organisations sont tombées dans le piège de recourir aux courriels comme moyen privilégié de 
communication, délaissant les rencontres en face à face et, ayant pour conséquence de limiter la coopération. Dans ce contexte où les zones communes de travail sont minimisées, l'organisation des relations de travail ne semble pas avoir bénéficié d'une réflexion fine sur la façon de faire coopérer les équipes de conception et les équipes d'encadrement, nuisant au bon fonctionnement des cours.

\section{BIBLIOGRAPHIE}

« Editorial : preparing teachers for tomorrow's technologies », Contemporary Issues in Technology and Teacher Education, 12(1), 2012, p. 1-5.

Anca Boboc et Thierry Taboy, « Numérique et transformations du monde du travail : vers de nouveaux équilibres ", Travail et numérique, 2014. Disponible en ligne : https://digital-societyforum.orange.com/fr/les-forums/267-

numerique_et_transformations_du_monde_du_travail__vers_de_nouveaux_equilibres, consulté le 4 avril 2017.

Anna Comas-Quinn, Beatriz de los Arcos et Raquel Mardomingo, «Virtual learning environments (VLEs) for distance language learning : Shifting tutor roles in a contested space for interaction », Computer Assisted Language Learning, 25(2), 2012, p. 129-143. Disponible en ligne : http:// oro.open.ac.uk/31505, consulté le 4 avril 2017.

Anne Mai Walder, «Les innovations technologiques sont-elles à l'origine des nouveaux rythmes et temporalités de l'université contemporaine ? ", Distances et médiations des savoirs, 16, 2016. Disponible en ligne : https://dms.revues.org/1605, consulté le 30 novembre 2017.

Chris Klisc, Tanya McGill et Valerie Hobbs, « The effect of instructor information provision on critical thinking in students using asynchronous online discussion », International Journal on ELearning, 11(3), 2012, p. 247-266.

Christian Depover et Jean-Jacques Quintin, « Tutorat et modèles de formation à distance », In Christian Depover, Bruno De Lièvre, Daniel Peraya, Jean-Jacques Quintin et Alain Jaillet, (dir.), Le tutorat en formation à distance, De Boeck, Bruxelles, 2011, p. 15-28.

Christian Depover, « La place et l'importance du tutorat dans les nouveaux dispositifs de formation à distance ", In Pierre-Jean Loiret, (dir.), Un détour par le futur. Les formations ouvertes et à distance à l'Agence universitaire de la Francophonie, Éditions des archives contemporaines, Paris, 2013, p. 69-82. Disponible en ligne : http://www.bibliotheque.auf.org, consulté le 4 avril 2017.

Christian Depover, Vassilis Komis et Thierry Karsenti, «Le contrôle de qualité : un outil indispensable pour asseoir la légitimité de la formation à distance ? ", Formation et profession, 20 (2), 2012, p. 1-8.

Christine Sarpentier, La communication du tuteur en formation à distance : étude de cas unique analysant l'attrait de l'utilisation de l'enseignement explicite comme support dans les interventions écrites effectuées par des tuteurs de la Téluq (mémoire de maîtrise, TéléUniversité, Québec, Canada), 2015. Disponible en ligne : http://r-libre.teluq.ca/810/1/ Sarpentier.pdf, consulté le 4 avril 2017. 
Chun-Wang Wei, Nian-Shing Chen et Kinshuk, « A model for social presence in online classrooms ", Educational Technology Research and Development, 60, 2012, p. 529-545.

Gee-Woo Bock et Young-Gul Kim, « Breaking the myths of rewards : an exploratory study of attitudes about knowledge sharing », Information Resources Management Journal, 15(2), 2002, p. 14-21.

Hiller A. Spires, Eric Wiebe, Carl A. Young, Karen Hollebrands et John K. Lee, « Toward a new learning ecology : professional development for teachers in $1: 1$ learning environments ", Contemporary Issues in Technology and Teacher Education, 12(2), 2012, p. 232-254.

Jean Loisier, Guide de communication éducative et de choix technologiques en formation à distance, Document préparé pour le Réseau d'enseignement francophone à distance du Canada (REFAD), 2009. Disponible sur http://www.refad.ca, consulté le 22 septembre 2015.

Jean-Claude Bertin et Jean-Paul Narcy-Combes, « Tutoring at a distance : modelling as a tool to control chaos », Computer Assisted Language Learning, 25(2), 2012, p. 111-127.

Joanne Kaattari, Guide des technologies et outils efficaces pour le perfectionnement et la formation en ligne du personnel, Community Literacy of Ontario, financé par Emploi Ontario, 2011.

John Nworie, Noela Haughton et Stephanie Oprandi, « Leadership in distance education : qualities and qualifications sought by higher education institutions ", American Journal of Distance Education , 26, 2012, p. 180-199.

John Short, Ederyn Williams et Bruce Christie, The social psychology of telecommunications, Wiley, New York, 1976.

John W. Creswell, Educational research : planning, conducting and evaluating quantitative and qualitative research, Pearson, Boston, 2012.

Maria Fernández-Toro et Concha Furnborough, « Feedback on feedback : eliciting learners' responses to written feedback through student-generated screencasts », Educational Media International, 51(1), 2014, p. 35-48.

Ming-Tien Tsai et Nai-Cheng Cheng, « Understanding knowledge sharing between IT professionals : an integration of social cognitive and social exchange theory ", Behaviour \& Information Technology, 31(11), 2012, p. 1069-1080.

Nicole Racette, Bruno Poellhuber et Marie-Pierre Bourdages-Sylvain, « Responsables de cours et tuteurs : un travail à distance? ", Revue internationale des technologies en pédagogie universitaire (RITPU), soumis en octobre 2017, 25 p.

Nicole Racette, Bruno Poellhuber et Marie-Pierre Bourdages-Sylvain, « Quelles sont les caractéristiques de l'emploi et du travail des tuteurs en formation ouverte et à distance ? ", Distances et médiations des savoirs, 18, 2017. Disponible en ligne : http://dms.revues.org/1835, consulté le 4 mai 2017.

Patrick Guillemet, Organisation et contexte québécois. Une perspective communicationnelle, Presses de l’Université du Québec, Québec, 1993.

Paulette Dilworth, Ana Donaldson, Marshall George, Don Knezek, Mike Searson, Kendall Starkweather, Marilyn Structchens, John Tillotson et Sharon Robinson,

Said Berrouk et Alain Jaillet, « Discours et impact de tuteurs en formation à distance », Études de linguistique appliquée, 160, 2010, p. 479-497. Disponible en ligne : https://www.cairn.info, consulté le 4 avril 2017. 
Sandrine Decamps et Christian Depover, « La perception du tutorat par les acteurs de la formation à distance ", In Christian Depover, Bruno De Lièvre, Daniel Peraya, Jean- Jacques Quintin et Alain Jaillet, (dir.), Le tutorat en formation à distance, De Boeck, Bruxelles, 2011, p. 109-124.

Sarita Jane Robinson, Luna C. Munoz Centifanti, Gayle Brewer et Lynda Holyoak, « The Benefits of Delivering Formative Feedback via Video-Casts », UCLan Journal of Pedagogic Research, 6(1), 2015. Disponible https://www.researchgate.net/ publication/283566579_The_Benefits_of_Delivering_Formative_Feedback_via_Video-Casts, consulté le 20 mars 2017.

Serge Casasus, Du meilleur usage des outils de communication numérique dans les entreprises, Document préparé pour l'Observatoire de la Responsabilité Sociétale des Entreprises, 2015.

Shenghua Zha et Christy Lee Ottendorfer, « Effects of peer-led online asynchronous discussion on undergraduate students'cognitive achievement », American Journal of Distance Education, 25, 2011, p. $238-253$.

Tae-Jun Cho, "The impact of types of interaction on student satisfaction in online courses ", International Journal on E-Learning, 10(2), 2010, p. 109-125.

Tomasz Filipowski, Przemyslaw Kazienko et Piotr Brodka, « Web-based knowledge exchange through social links in the workplace ", Behaviour and Information Technology, 31(8), 2012, p. 779-790.

Vivianne Glikman, « Pédagogies et publics des formations à distance. Quelques touches historiques », Distances et médiations des savoirs, 8, 2014. Disponible en ligne : https:// dms.revues.org/902, consulté le 30 novembre 2017.

\section{RÉSUMÉS}

Dans une recherche menée dans quatre établissements d'enseignement, nous abordons la question suivante : comment le numérique contribue- $t$-il ou limite-t-il la coopération entre les équipes de conception et les équipes d'encadrement ? À partir de 44 entrevues individuelles et 8 entrevues de groupes, les résultats montrent que ces équipes, éloignées physiquement, se rencontrent très peu et utilisent majoritairement le courriel pour communiquer, outil que nous considérons permettre surtout le transfert des connaissances plutôt que les échanges, et ce, à partir des caractéristiques rapportées par les répondants. Ainsi, lorsque les courriels sont utilisés pour échanger, ils sont susceptibles d'engendrer des dysfonctionnements dans les relations. Les outils d'échange (forums, visioconférences, etc.) sont très peu utilisés. L'emploi des outils de développement des connaissances (logiciels de formation, etc.) est fortement souhaité par les tuteurs répondants, bien que ces derniers réclament surtout des rencontres en face à face pour créer d'abord une relation, afin de permettre de mieux coopérer et améliorer ainsi la qualité de leur travail.

In a research project conducted in four educational institutions, we addressed the following question: How does digital technology enhance or limit cooperation between the design and management teams? From 44 individual interviews and 8 group interviews, the results show that these teams, being physically distant, seldom meet and mainly use email for communicating, which we describe as a tool for transferring knowledge rather than one for communicating between parties, and this, from the characteristics reported by the respondents. Thus, when emails are used to communicate, they are likely to cause dysfunctions in the relationships. 
Communication tools (forums, videoconferences, etc.) are very seldom used. The use of tools for knowledge development (training software, etc.) is strongly desired by the responding tutors, although they mainly call for face-to-face meetings to first create a relationship, in order to better cooperate and thus improve the quality of their work.

En una investigación llevada a cabo en cuatro establecimientos educativos hemos abordado la siguiente pregunta: ¿cómo las tecnologías digitales contribuyen o limitan la cooperación entre los equipos de diseño pedagógico y los equipos de tutoría? A partir de 44 entrevistas individuales y 8 entrevistas grupales, los resultados muestran que estos equipos, separados físicamente, se reúnen muy poco y para comunicarse utilizan predominantemente el correo electrónico. Según las características señaladas por los participantes consideramos que, más que la interacción, esta herramienta favorece la transmisión de conocimiento. Así, cuando los mensajes por correo electrónico son utilizados para dialogar, pueden engendrar relaciones disfuncionales entre quienes participan del intercambio. Las herramientas de interacción (foros, videoconferencias, etc.) son poco utilizadas. Los tutores participantes en el estudio solicitan el uso de herramientas para el desarrollo de conocimientos (software educativo, etc.) y reclaman encuentros presenciales para crear una relación que permita una mejor cooperación y el mejoramiento de la calidad de su trabajo.

\section{INDEX}

Mots-clés : coopération, numérique, formation à distance, communication, courriels

Palabras claves : cooperación, tecnologías digitales, educación a distancia, comunicación, correo electrónico

Keywords : cooperation, digital, distance learning, communication, emails

\section{AUTEURS}

\section{NICOLE RACETTE}

Téluq, Canada

MARIE-PIERRE BOURDAGES-SYLVAIN

Téluq, Canada 